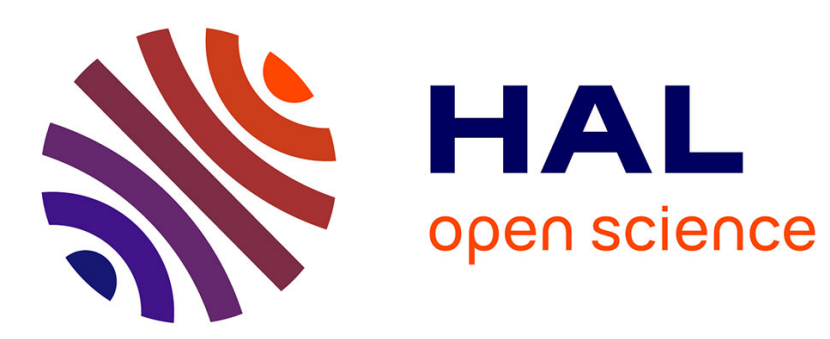

\title{
An optimization model for selecting a product family and designing its supply chain
}

Jacques Lamothe, Khaled Hadj-Hamou, Michel Aldanondo

\section{To cite this version:}

Jacques Lamothe, Khaled Hadj-Hamou, Michel Aldanondo. An optimization model for selecting a product family and designing its supply chain. European Journal of Operational Research, 2006, 169 (3), pp.1030-1047. 10.1016/j.ejor.2005.02.007 . hal-00452961

\section{HAL Id: hal-00452961 https://hal.science/hal-00452961}

Submitted on 6 Nov 2018

HAL is a multi-disciplinary open access archive for the deposit and dissemination of scientific research documents, whether they are published or not. The documents may come from teaching and research institutions in France or abroad, or from public or private research centers.
L'archive ouverte pluridisciplinaire HAL, est destinée au dépôt et à la diffusion de documents scientifiques de niveau recherche, publiés ou non, émanant des établissements d'enseignement et de recherche français ou étrangers, des laboratoires publics ou privés. 


\title{
An optimization model for selecting a product family and designing its supply chain
}

\author{
Jacques Lamothe *, Khaled Hadj-Hamou, Michel Aldanondo \\ Industrial Engineering Center, Ecole des Mines d'Albi-Carmaux, Campus Jarlard, Route de Teillet, 81013 Albi CT Cedex 09, France
}

\begin{abstract}
When designing a new family of products, designers and manufacturers must define the product family and its supply chain simultaneously. At the very first step of the design process, designers propose various solutions for the set of variants of a product family and their bill-of-materials. The second step is to select some of these variants while choosing the architecture of the supply chain. A mixed integer linear programming model is investigated that optimizes the operating cost of the resulting supply chain while choosing the product variants. This work is applied to the problem of an automotive supplier.
\end{abstract}

Keywords: Supply chain management; Product family design; Supply chain design; Mixed integer linear programming (MILP)

\section{Introduction}

Nowadays, the growing demand for customizable or configurable products involves an increasing number of product variants and a growing complexity of products while controlling the product costs and the customer lead-time. This task becomes more difficult when the supply chain layout has a significant influence on operating costs. Consequently, when designing a new product family, a consistent approach is necessary to quickly define a set of product variants and their relevant supply chain, in order to guarantee the customer satisfaction and to minimize the total operating cost of the global supply chain.

This paper proposes a design approach that allows to define simultaneously a product family and its supply chain while facing a customer demand with a large diversity. Between "Product Design" and "Supply

\footnotetext{
${ }^{*}$ Corresponding author. Tel.: +33 5634932 16; fax: +33563493183.

E-mail addresses: jacques.lamothe@enstimac.fr (J. Lamothe), khaled.hadjhamou@enstimac.fr (K. Hadj-Hamou), michel. aldanondo@enstimac.fr (M. Aldanondo).
} 
Chain Management", our simultaneous approach is closely related to the field of "Concurrent Engineering" [25] and more accurately to a "Design For $X(D F X)$ " approach [15], where $X$ is the Supply Chain.

Design For Supply Chain (DFSC) or Design For Logistics (DFL) basics have been defined by Lee in $[16,17]$. These works underlined various interests in adapting the design of a product family in order to enhance the costs and lead-time of a given supply chain. They mainly quantify how the manufactured diversity can be decreased and the consequences on safety stocks cut down thanks to the following means: various kinds of postponement of a product variant (time, place or form postponement) [17,18], product and process modularization or standardization [7], operation reversals that cause a component reversal in the bills-of-materials [19]. More recently, Van Hoek [26] synthesized the developments of postponement applied to a supply chain. Anderson [1] and Pine II [24] also underlined the integration of these concepts in order to propose mass-customizable products.

Our purpose is to help the designers of a product family in making design choices and evaluating the consequences of their choices on the layout of the supply chain that will deliver the products.

Therefore, we consider a two-step iterative process (Fig. 1):

- The first step, the bill-of-materials provider step, easily and quickly defines error free bill-of-materials for a set of pre-identified customer requirements. It is a pure product design process. It is only considered at the preliminary design step that deals with some design principles and product architectures. It generates many product variants so that a selection can be made in the second step.

- The second step optimizes the compromise "over equipment cost/reference management cost", through the simultaneous selection of (i) the supply chain (where to manufacture, to assemble and to store) and (ii) the product variants of the product family.

The process is interactive because knowing the product variants selected at the second step can help the product designers in adjusting, during the first step, the design principles and the set of customer requirements.

Our purpose in this paper is not to explain how to handle the first step. But an example of a tool for supporting the bill-of-materials process using the analogy with a configuration process can be found in [14]. We focus on the second step, and therefore it is necessary to specify:

- how to depict, through a generic bill-of-materials, the product diversity resulting from the first step (Section 2);

- the model that enables to make the various choices (Section 3).

Finally an application dealing with the design of a wiring harness family for a car manufacturer will be depicted in Section 4.

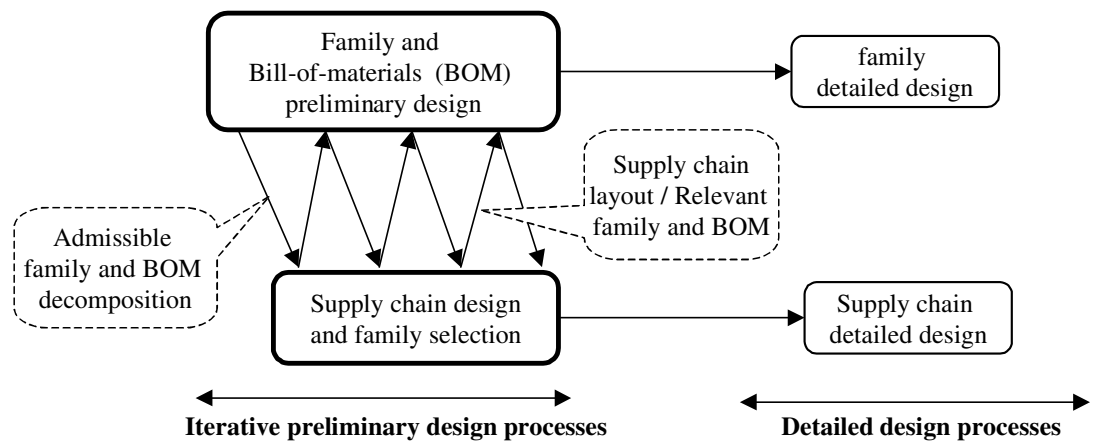

Fig. 1. A two-step iterative process for product family and supply chain design. 


\section{Defining the generic bill-of-materials}

\subsection{Modeling the product and design diversities}

Demand diversity can be considered from various points of view: the customer or functional one, the product or physical one, and the supply chain or process one.

From the functional point of view, the diversity refers to the set of requirements that a customer can express. Each requirement is derived in a series of ordered service levels (service level $1 \prec$ service level $2 \prec \ldots \prec$ service level $n$ ). These service levels distinguish levels of complexity and cost in order to obtain the function. For example, the service levels of the function "car window lifter" can vary from "manual lifter" up to "electrical lifter with alarm and pinch protection". Consequently, the customer diversity comes from the combinatory gathering all the service levels relevant to the multiple requirements: the set $\{($ requirement, service level)\} defines the market needs that must be covered by the product family.

From the product point of view, a product family is a set of physical product variants and is defined in order to fulfill the market needs. A product variant fulfills all of the requirements with a given specific service level. Moreover, a product variant is split up into components based on its bill-of-materials. The physical diversity usually refers to the cardinal of this product family or to the cardinal of the set of components. As we consider an order relation between the service levels of each requirement, a partial order relation also exists between the product variants within a product family: a Variant V1 is greater than a Variant V2, if, for each requirement, the service level of V1 is greater than the service level of V2.

Now, let us consider a given customer demand defined by a set of service levels, one per functional requirement: demand $=\{($ requirement, demanded service level $)\}$. There may not exist in the product family a product that exactly matches the demanded service levels. But the interest of the producer remains to satisfy this demand at the lowest cost and thus as close as possible to the demanded service levels. Consequently, the demand will be fulfilled by the smallest variant (in the sense of the partial relation of order) that over-satisfies all the demanded service levels. Another consequence is that a product family must at least contain one variant that exactly matches all the functional requirements at the maximal service level so that any demand can be over-satisfied by this variant. This means that it is not necessary to manufacture all the possible variants: one can select the variants to manufacture in order to satisfy all the functional diversity at the lowest global cost (or at the maximal profit).

Fig. 2 depicts the design challenge in a case with 2 requirements and 3 service levels. There can be 9 variants (Variant $x \cdot y$ satisfies Requirement 1 at the Service Levels $1 / x$ and Requirement 2 at the Service Levels $2 / y$ ). In practice, it is not possible to manufacture all the variants because of the combinatory size

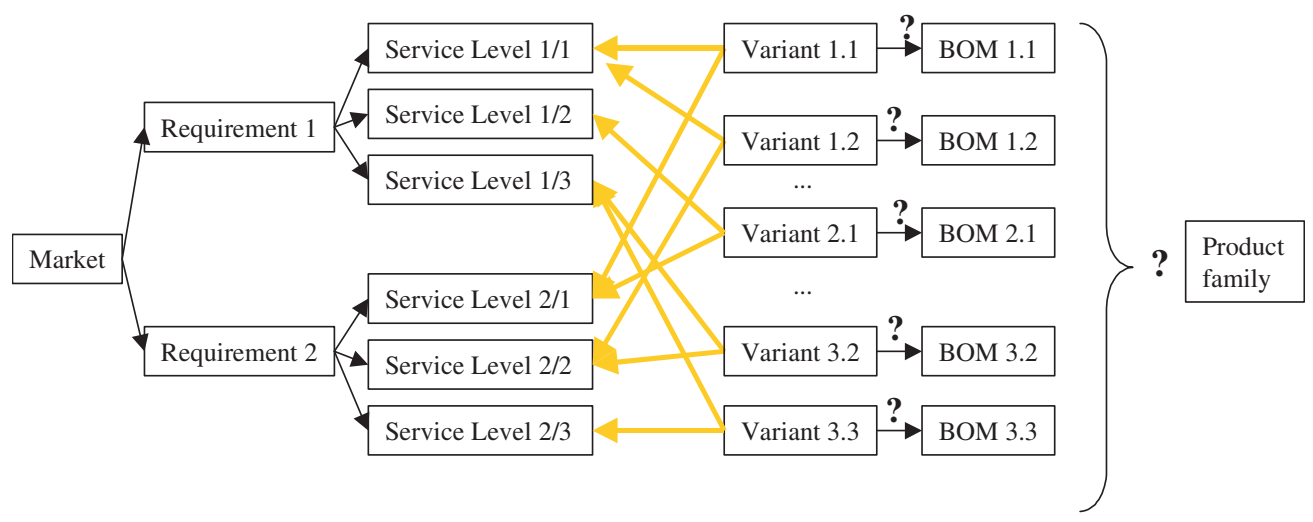

Fig. 2. The design challenge. 
( $V$ requirements with $W$ service levels lead $W^{V}$ variants). But the Variant 3.3 is necessary because it enables to over-satisfy any demand. So the design challenge is to select some of these variants and design their billof-materials.

The design diversity is therefore the diversity of choices in order to define the set of variants, their associated service levels, and their bill-of-materials. The systematic approach defined by Pahl and Beitz [22] is usually adopted for designing a product. The first step consists in choosing design principles. These design principles are technical and technological choices and the definition of a product family architecture. The architecture defines the types of sub-assemblies, their technology, and their relations.

In order to design the architecture of a product family two main strategies are identified which we call "market-segment oriented" strategy and "modular" strategy. Next sub-sections detail how to describe the result of such design strategies.

\subsubsection{Market segment design strategy}

The market segment strategy works on a restricted list of market segments usually defined by a marketing department. A market segment is characterized by fixing a specific service level for each of the functional requirements. Then a variant is designed in order to fulfill a specified market segment. As several design principles can be applied to the same market segment, several admissible variants can be obtained per market segment. Moreover, as market segments are defined as a set of service levels of the requirements, a partial order relation between the market segments also exists.

In the case of the example with 2 requirements and 3 service levels, Fig. 3 depicts the result of a design process in which only 4 market segments have been selected among the 9 possible ones. Several design principles have been applied to the market segments MS1 (3 principles) and MS4 (2 principles), and corresponding variants are obtained (notation: Variant $V-i$ denotes the design principle number " $i$ " for the variant “ $V$ ').

The bold arrows depict the order relations on the market segments. Therefore a variant designed for the market segment MS2 can also over-satisfy the needs of MS1.

Demand will always be expressed on the four market segments (MS1, .., MS4). But the design choices that remain finally are:

- whether to over-satisfy a market segment with a variant designed for an upper market segment;

- to select a variant among the designed ones for the not over-satisfied market segments.

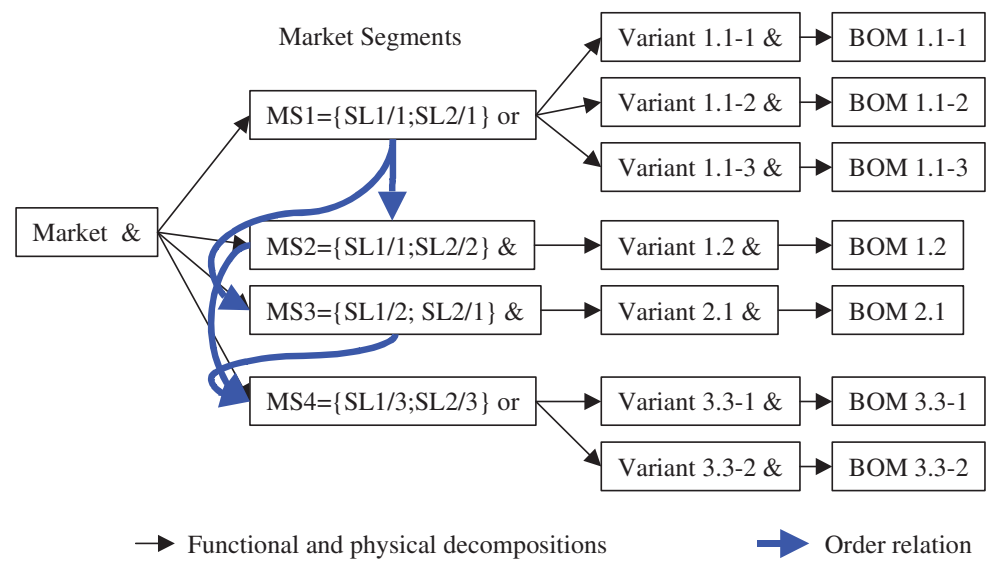

Fig. 3. Result of a bill-of-materials generation process throughout a market segment strategy. 
In the example, an extreme decision can be to only manufacture either Variant 3.3-1 or Variant 3.3-2 in order to satisfy all the market segments.

\subsubsection{Modular design strategy}

The second strategy aims at adopting modular principles. This strategy appears to be essential in order to design mass-customizable products $[1,7,24]$. The principle is to design at least one module variant per functional requirement and per service level so that any demand can be fulfilled with the assembly of the desired module variants. This modular principle forces: (i) to design a standard platform on which any modular variant can be assembled; (ii) to define standard interfaces between the module variants and the standard platform.

Moreover, modules are supposed to be independent so that many design principles can be applied to a same service level and thus many equivalent module variants are obtained.

Fig. 4 depicts the result of a modular strategy for the same 2 requirements and 3 service levels example. In that case, 2 different design principles have been applied to the requirement 2 with the service level 2 (notation: Module $V / W-i$ denotes the design principle number " $i$ " for the Requirement $V$ with a service level $W$ ).

The bold arrows represent the order relation on the service levels. Therefore the module $1 / 2$ designed for the service level SL1/2 can also over-satisfy the needs of SL1/1.

Demand will always be expressed on the six service levels (SL1/1, ., SL2/3). But the design choices that remain finally are:

- whether to over-satisfy a service level with a module designed for a higher service level;

- to select a module among the designed ones for the not over-satisfied service levels.

In the example, an extreme decision can be to only manufacture modules $1 / 3$ and $2 / 3$ in order to satisfy all the market segments.

\subsubsection{Mixed market segment and modular design strategies}

Market segment and modular design strategies can be mixed in order to reach a compromise between over-equipment costs, over-satisfaction costs and management costs.

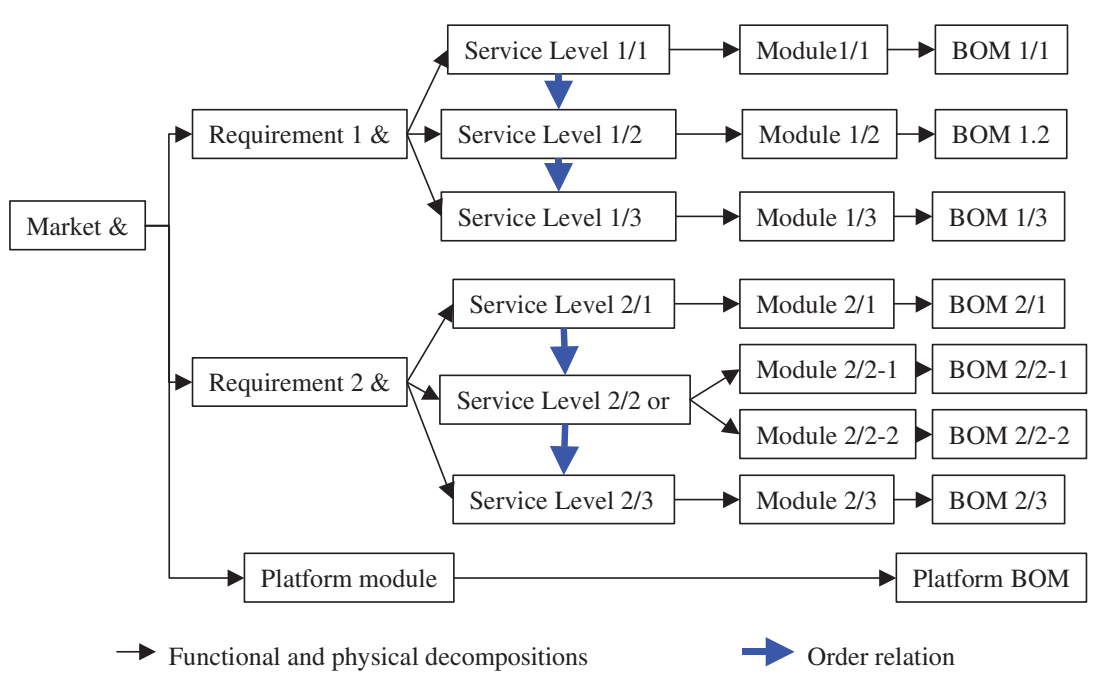

Fig. 4. Example of result of a bill-of-materials generation process throughout a modular strategy. 
In a market segment oriented strategy, some market segments have common service levels for several requirements. A designer may be interested in developing variants for these market segments that share common modular components.

Reciprocally, during a modular design strategy a designer can find cheaper to design a module that simultaneously fulfills a subset of functional requirements. Therefore, the designer should define market segments and thus module variants for this subset of customer requirements.

These approaches can be frequently observed in various markets. For instance, household electrical appliance families are usually decomposed into market segments; personal computer families are much more modular; while automotive families come from a compromise between market segment and modular approaches: a car is usually defined according to a market range level, such as bottom, middle and luxury but also sport and touring, plus some optional requirements (color, road map computer, loudspeaker, motor).

Fig. 5 is an example of a mixed design strategy of the examples of Figs. 3 and 4 . The market segment MS1, MS2 and MS3 have been gathered within a single market segment MS123 which is defined throughout a modular approach. This approach allows to fulfill a demand gathering $\{$ (Requirement 1 , Service Level 1/2); (Requirement 2, Service Level 2/2)\} that was not offered in Fig. 3.

The design choices that remain finally are:

- whether to over-satisfy MS123 with a variant designed for MS4;

- whether to over-satisfy SL1/1 or SL2/1 with a module designed for SL1/2 or SL2/2 if MS123 is not oversatisfied;

- to select a module or a variant among the designed ones for the not over-satisfied service levels or market-segments.

\subsubsection{Consequences on product diversity: Two decisions}

The result of the bill-of-materials design process appears to be a tree decomposition of the market needs (throughout the unbold arrows).

The nodes of the upper part of the tree identifies subsets of couples (requirement, service levels). These nodes correspond with either market segments, or requirements or couples (requirement, service level). They depict the functional offer that is proposed to the customers.

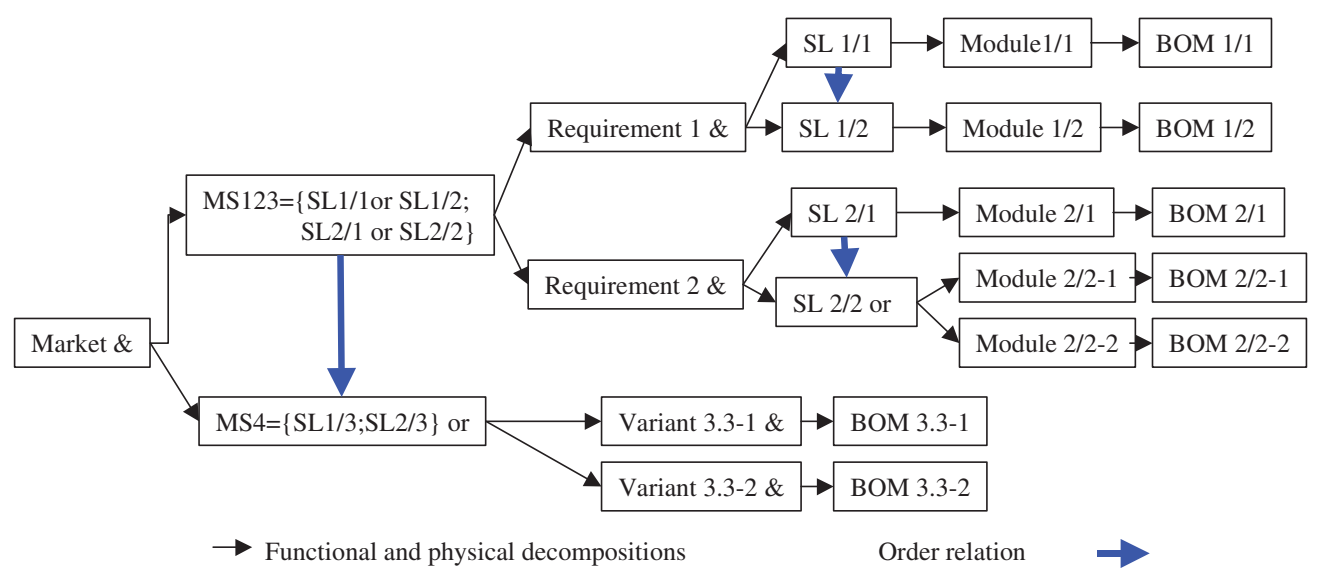

Fig. 5. Example of result of a mixed design strategy. 
The leafs of this tree are the bill-of-materials (BOM), that correspond with either a module or a variant. A module is the result of a design principle matching a service level of a requirement. A variant is the result of a design principle matching a market segment. They depict the technical solution that matches the previous functional offer.

Moreover, from the original order relation between the service levels of the functional requirements, order relations between market segments or service levels (bold arrows) appear. Looking for example at the link between MS123 $\rightarrow$ MS4 in Fig. 5, the order relation expresses: if necessary a variant designed for the market segment MS4 can fulfill the market segment MS123.

Consequently, two types of manufacturing decisions must be taken:

- using the order relation between market segments or service levels, one must choose whether some will be over-satisfied and therefore the corresponding variants or modules will not be manufactured;

- when several design principles have been applied, one must choose one of the resulting bill-of-materials.

\subsection{The generic bill-of-materials (G-BOM)}

During a planning process, the bill-of-materials is used in order to compute net requirement of items given the demand of final products. Here, a generic bill-of-materials (G-BOM) expresses the admissible ways to distribute the market needs into the designed elements.

The G-BOM is a directed tree, in which nodes refer to items and arc values refer to the necessary quantity of child item per parent item. To match the extension of a classical bill-of-materials, the key idea is to add new notions of "logical item" versus "physical item" and "exclusive OR" node versus usual "AND" node.

- An "exclusive OR" node is introduced to show that one and only one item must be selected among all the child items of the node. It allows representing the choice of existence of a child item. An "AND" node expresses that all its child items must be gathered in order to make it.

- A logical item is only necessary for the G-BOM expression. It comes from the product design approach that has been taken. Due to its logical meaning, it can neither be manufactured, nor stored, nor shipped. During a planning process, it allows to compute a net required quantity coming from its parent items that must be fulfilled by child items. Conversely, a physical item can be stored, manufactured or shipped. A logical item is mostly used in order to formalize either the market, or a subset of requirements, or the result of a choice between design and over-satisfaction alternatives.

- An arc value is usually 1 except when it expresses a market segmentation of the requirement of the parent item.

Fig. 6 shows the generic bill-of-materials corresponding to the result of the product design process depicted in Fig. 5. The "Variants", "Module" and "BOM" items are physical as they can be manufactured if they are selected. The "Sub-assembly" item has been added. It depicts the assembly of modular components and therefore is also physical.

The values on the arcs between "Market" and the market segments items ("MS123" or "MS4") express an estimated market share between market segments. Those between requirement items ("Req1" for example) and the service level items ("SL1/1" or "SL1/2") express estimated demand decomposition on the service levels.

Therefore, when the "OR" choices are made one can express how a market volume induces net requirements on the selected BOM articles. Note that the "OR" choices are supposed to be hidden from the 


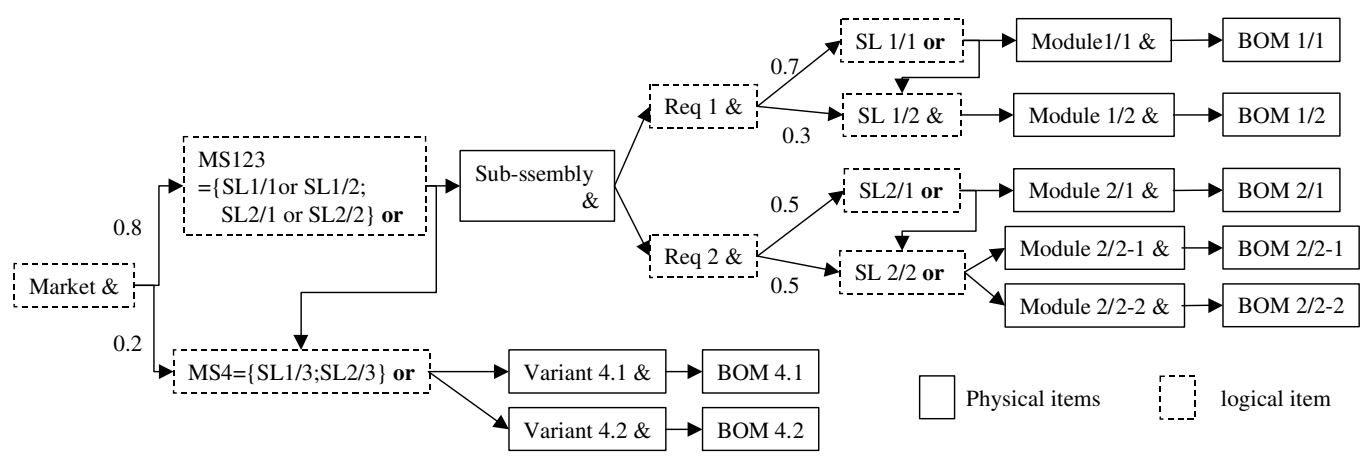

Fig. 6. A generic bill-of-materials.

customers and consequently do not change the functional diversity offered. So, the market shares do not depend on the choices made.

The goal of the model of the next section is to make these "OR" choices while designing a supply chain.

\section{Supply chain and product family optimization}

In this section, we define a MILP (mixed integer linear programming) model that optimizes the supply chain design, processes a generic bill-of-materials (G-BOM) and therefore identifies the product family. We therefore define a tool that supports the second step of our global approach (see Fig. 1).

Among the works dealing with supply chain design $[5,11,12]$, strategic planning is interested in optimizing the layout of a complete supply chain. Basically, the strategic design of a supply chain decides about or quantifies (i) the existence, location and capacity of facilities, (ii) the shipping channels to use and (iii) the product flows in order to minimize the supply chain total costs.

Many models have been formulated for this problem, called GSCM (Global Supply Chain Model). One of the earliest papers [8] presents an algorithm based on Benders decomposition to solve a multi-articles single-period production-distribution problem. This work has been expanded by $[3,9,10]$. More recently, a more comprehensive models to optimize single-country supply chains was developed [6].

Other models introduce extensions in order to consider: the effect of taxes, duties, exchange prices that force to optimize the global total profit instead of the total costs [2,5]; scale economies on manufacturing or inventory systems [21]; the choice of technological manufacturing systems [23]; the effect of uncertainties on demand or on exchange rates [13,20].

Basically, the global supply chain design models $[2,4,11,12,27]$ are defined with:

- continuous variables: one per item, time period, facility with three kinds of activities (manufacturing, inventory and shipping);

- binary and integer variables: one per facility describing whether the facility is used or not, and the number of resource lines opened;

- classical strategic planning linear constraints between theses variables;

- a cost function to minimize, that gathers variable costs (manufacturing, inventory and shipping) and fixed costs (facilities and resource lines fixed costs).

Here, a classical GSCM is extended to consider the G-BOM. Many of the previous extensions could certainly be integrated to this model (especially taxes and duties). But, for simplicity, this paper concentrates on the G-BOM integration with a classical GSCM model. 
The resolution will provide (i) the product family retained, (ii) the list of opened facilities and resource lines and (iii) the optimal supply chain operating cost.

Therefore, let us add, for representing the generic bill-of-materials:

- binary variables: one for each item of the G-BOM describing whether the item exists or not, one for each G-BOM link "parent $(\mathrm{OR}) \rightarrow$ child" (arising only with the "OR" nodes) describing if the parent item requires or not the child item;

- constraints between these binary variables.

A mixed integer linear programming model is defined in the following as an extension of basic models such as: (i) a single shipping channel is available between any two facilities; (ii) manufacturing or shipping activities have much smaller lead-times than the time period, and thus are supposed continuous.

Literally, the model can be expressed as:

Minimize total cost $=$ Fixed costs relevant to the existence of items, facilities and resource lines and shipping channels (1) + Variable costs relevant to manufacturing, inventory and shipping (2).

We assume that the market shares do not depend on the choices made on the G-BOM. Moreover taxes, duties and exchange rates are not considered. Otherwise, the objective would have been to maximize the total profit.

Subject to constraints:

- Generic bill-of-materials constraints (constraints (3)-(9)).

- Item flow conservation constraints (constraints (10)-(13)).

- Shipping constraints (constraints (14) and (15)).

- Inventory constraints (constraints (16) and (17)).

- Capacity constraints (constraints (18)).

- Integrality constraints (constraints (19)).

But before detailing the model, let us depict the notations and variables.

\subsection{Notations of sets, costs and parameters}

The various sets used in the model:

$P$ is the set of items of the generic bill-of-materials, indexed by $p$ or $q$,

$\Phi$ is the set of physical items, indexed by $p$ or $q$,

$\bar{\Phi}$ is the set of logical items, indexed by $p$ or $q$,

$B O M_{p}$ is the set of child items of an item $p$ (with "AND" or "OR" node),

$B O M_{p}^{-1}$ is the set of parent items of an item $p$,

$P_{c}$ is the sub-set of items with external demand relevant to customer $c$, indexed by $p$ or $q$,

$P^{\wedge}$ is the set of items with an "AND" node, indexed by $p$ or $q$,

$P^{\vee}$ is the set of items with an "OR" node, indexed by $p$ or $q$,

$T$ is the set of time periods, indexed by $t$,

$C$ is the set of customers, indexed by $c$,

$U$ is the set of production/inventory facilities, indexed by $u$ or $v$, 
$R$ is the set of manufacturing resource types, indexed by $r$,

$P_{r}$ is the set of physical items manufactured on the resource type $r$, indexed by $p$ or $q$.

The fixed and variable costs of items, facilities and shipping channels:

$E C F_{p}$ is the fixed cost of existence of a physical item $p$,

$U C F O_{u}$ is the fixed cost of opening facility $u \in U$,

$U C F O_{r u}$ is the fixed cost of opening a line $r$ at facility $u \in U$,

$M C F_{u v}$ is the fixed cost of a shipping channel from facility $u \in U$ to $v \in U \cup C$,

$U C V_{p u}$ (resp. $S C V_{p u}$ ) is the variable cost to make (resp. store) an item $p$ at facility $u \in U$,

$M C V_{p u v}$ is the variable cost of shipping one item $p$ between facilities $u \in U$ and $v \in U \cup C$.

Parameters:

$D$ is the duration of a time period,

$M_{\infty}$ is the maximum number of items to manufacture, to store or to ship on a time period,

$D_{e m} m_{p t}$ is the demand for item $p$ from customer facility $c$ during period $t$,

$\alpha_{p q}$ is the quantity of child item $p$ required to make one unit of parent item $q$,

$\operatorname{Max} L P_{r u}$ is the maximum number of resource lines of type $r$ at facility $u$,

URes $_{r p}$ is the time of a resource line of type $r$ necessary per product $p \in P_{r}$,

$C f_{p u}$ (resp. $C t_{p u v}$ ) is the manufacturing (resp. shipping) lead-time of product $p$ at facility $u \in U$ (resp. up to facility $v \in U \cup C$ ) defined as a percentage of $D$,

$\operatorname{Cov}_{p u}$ is the percentage of manufacturing and shipping lead-time of product $p$ to get at facility $u \in U$ as safety stock.

\subsection{Binary/continuous decision variables}

Binary variables:

$\lambda_{p}=1$ if item $p$ exists, otherwise 0,

$X_{u}=1$ if facility $u$ is opened, otherwise 0 ,

$\lambda_{p q}=1$ if item $q \in P^{\vee}$ requires item $p$ as a child, otherwise 0 . This binary variable selects a bill-of-materials link for item $q$ with an "OR" node.

$Z_{u v}=1$ if the shipping channel between facilities $u$ and $v$ exists, otherwise 0 ,

$L P O_{r u} \in N$ is the integer number of resource lines of type $r$ opened at facility $u$,

Continuous variables:

$X_{\text {put }}$ is the amount of net requirement associated to an item $p$ (physical or logical) at facility $u$ during time period $t$. For a physical item, this net requirement is also the quantity manufactured,

$X_{p q u t}$ is the amount of net requirement associated to a parent item $q$ with node "OR" using a child item $p$ at facility $u$ during period $t$, if the link $q \rightarrow p$ exists $\left(\lambda_{p q}=1\right)$,

$Y_{p u t}$ is the amount of a physical item $p$ stored at facility $u$ at the end of time period $t$,

$Z_{p u v t}$ is the amount of physical item $p$ shipped from facility $u$ to facility $v$ during period $t$,

Using these notations and decision variables, the mathematical programming model can be formulated. 


\subsection{Mathematical formulation}

\subsubsection{The objective}

The model minimizes the sum of total costs: fixed cost relevant to the items (1.1), plus facility, resource lines, shipping channel existences (1.2) plus variable manufacturing, inventory and shipping costs (2).

$$
\begin{aligned}
& \sum_{p}^{\Phi} E C F_{p} \cdot \lambda_{p}, \\
& +\sum_{u}^{U} U C F O_{u} \cdot X_{u}+\sum_{u}^{U} \sum_{v}^{U \cup C} M C F_{u v} \cdot Z_{u v}+\sum_{r}^{R} \sum_{u}^{U} U C F O_{r u} \cdot L P O_{r u}, \\
& +\sum_{p}^{\Phi} \sum_{u}^{U} \sum_{t}^{T}\left[U C V_{p u} \cdot X_{p u t}+S C V_{p u} \cdot Y_{p u t}+\sum_{v \neq u}^{U \cup C} M C V_{p u v} \cdot Z_{p u v t}\right] .
\end{aligned}
$$

\subsubsection{Generic bill-of-materials constraints}

The G-BOM constraints are introduced to express the existence of items and links of the G-BOM. According to the type of the G-BOM node ("AND" or "OR"), different constraints restrict the binary variables $\lambda_{p}$. Recall that variables $\lambda_{p q}$ are only defined if item $q$ corresponds to an "OR" node in the G-BOM.

3.3.2.1. For any kind of node. Constraints (3) require that a net requirement is associated to an item $p$ at facility $u$ if and only if this item exists and the relevant facility is opened. Constraint (4) expresses that when an item $p$ (without external demand) is selected, either one of its parent items with an "AND" node exists, or a link relevant to an "OR" node exists.

$$
\begin{aligned}
& X_{p u t} \leqslant M_{\infty} \cdot \lambda_{p} \text { and } X_{p u t} \leqslant M_{\infty} \cdot X_{u} \quad \forall p \in P, u \in U \cup C, t \in T, \\
& \lambda_{p} \leqslant \sum_{q}^{B O M_{p}^{-1} \cap P^{\wedge}} \lambda_{q}+\sum_{q}^{B O M_{p}^{-1} \cap P^{\vee}} \lambda_{p q} \quad \forall p \in P-P_{c} .
\end{aligned}
$$

3.3.2.2. For "OR" nodes. Constraints (5)-(8) are only relevant for items $q$ with an "OR" node ( $\left.q \in P^{\vee}\right)$. Constraint (5) ensures that a link relevant to an "OR" node exists if, and only if, both parent and child items exist. Constraint (6) stands that if an item $q \in P^{\vee}$ exists, one and only one link must be selected between $q$ and its child items. Constraint (7) means that the existence of a net requirement of an item $q$ triggers a gross requirement on its child items. Constraint (8) makes sure that if a link $q \rightarrow p$ exists, the net requirement associated to the item $q$ requires the child item $p$.

$$
\begin{aligned}
& \lambda_{p q} \leqslant \lambda_{q} \quad \text { and } \quad \lambda_{p q} \leqslant \lambda_{p} \quad \forall q \in P^{\vee}, p \in B O M_{q}, \\
& \sum_{p}^{B O M_{q}} \lambda_{p q}=\lambda_{q} \quad \forall q \in P^{\vee}, \\
& \sum_{p}^{B O M_{q}} X_{p q u t}=X_{q u t} \quad \forall q \in P^{\vee}, u \in U, t \in T,
\end{aligned}
$$




$$
X_{p q u t} \leqslant M_{\infty} \cdot \lambda_{p q} \quad \forall q \in P^{\vee}, p \in B O M_{q}, u \in U, t \in T .
$$

Constraints (5)-(8), altogether, imply that:

$$
X_{p q u t}=X_{q u t} \Longleftrightarrow \lambda_{p q}=1 \quad \forall q \in P^{\vee}, p \in B O M_{q}, u \in U, t \in T .
$$

3.3.2.3. For " $A N D$ " nodes. Constraint (9) is given only for an item $q$ with an "AND" node. It ensures that the existence of a parent item $q \in P^{\wedge}$ implies the existence of all its child items.

$$
\lambda_{p} \geqslant \lambda_{q} \quad \forall q \in P^{\wedge}, p \in B O M_{q} .
$$

\subsubsection{Flow conservation constraints}

Flow conservation constraints specify that for each facility $u$ and during each time period $t$, the inventory variation of an item $p$ must be equal to the sum of quantities generated in the facility $(X)$ and coming from other facilities $(Z)$ minus the sum of quantities shipped to other facilities ( $Z$ and $D e m$ ) and minus the sum of quantities consumed in the facility to satisfy the gross requirements associated to the parent items $(X)$.

According to our G-BOM definition: (i) a logical item can neither be manufactured, stored nor shipped, but it can generate a gross requirement in any facility, (ii) a physical item can neither be manufactured nor stored in a customer facility, but it can be shipped to it.

Therefore, according to the type of facility (manufacturing or customer), and the type of item (physical or logical), the flow conservation changes (see Fig. 7).

So, flow constraints are formulated for each case:

- Constraints (10): physical items in production facility,

$$
\begin{aligned}
& \forall p \in \Phi, u \in U, t \in T \\
& Y_{p u t}-Y_{p u t-1}=X_{p u t}+\sum_{v \neq u}^{U} Z_{p v u t}-\sum_{v \neq u}^{U \cup C} Z_{p u v t}-\sum_{q}^{P^{\vee} \cap B O M_{p}^{-1}} \alpha_{p q} \cdot X_{p q u t}-\sum_{q}^{P^{\wedge} \cap B O M_{p}^{-1}} \alpha_{p q} \cdot X_{q u t} .
\end{aligned}
$$

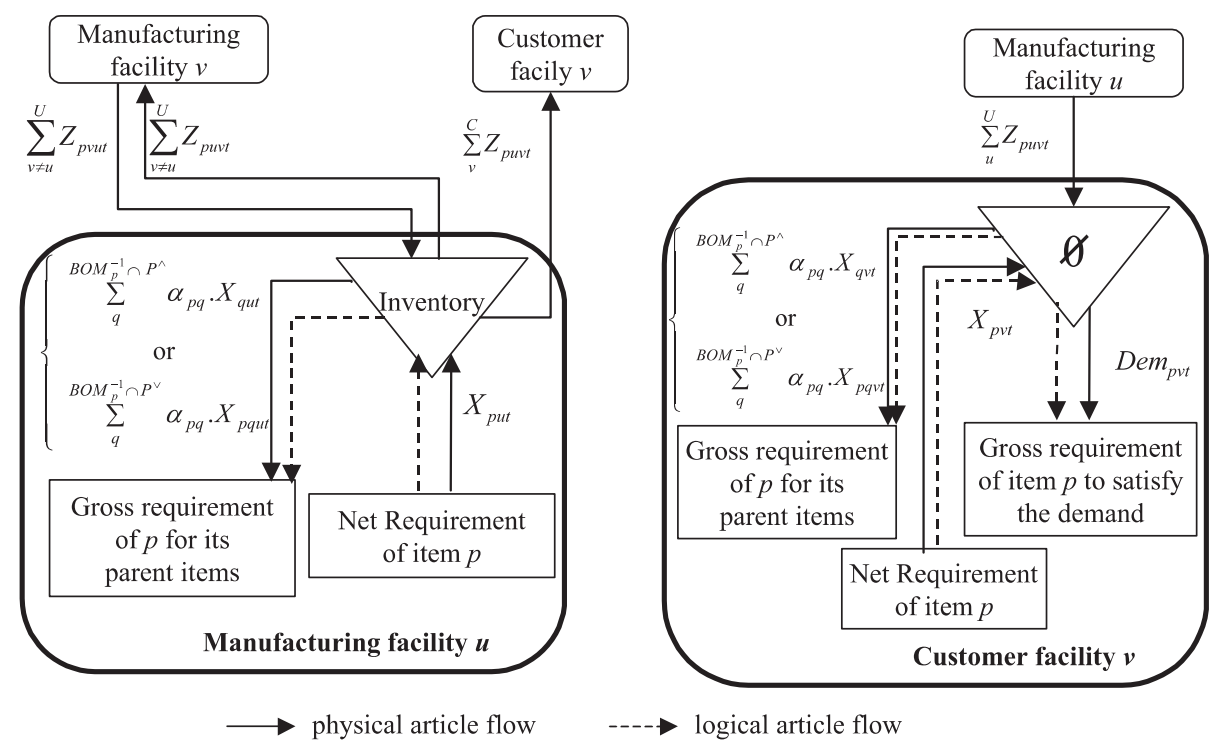

Fig. 7. Physical/logical item flows through a manufacturing or a customer facility. 
- Constraints (11): logical items in production facility,

$$
\begin{aligned}
& \forall p \in \bar{\Phi}, u \in U, t \in T \\
& 0=X_{p u t}-\sum_{q}^{\left(P^{\vee} \cap B O M_{p}^{-1}\right)} \alpha_{p q} \cdot X_{p q u t}-\sum_{q}^{\left(P^{\wedge} \cap B O M_{p}^{-1}\right)} \alpha_{p q} \cdot X_{q u t} .
\end{aligned}
$$

- Constraints (12): physical items in customer facility,

$\forall p \in \Phi, c \in C, t \in T$

$$
\operatorname{Dem}_{p c t}=\sum_{v}^{U} Z_{p v c t}-\sum_{q}^{P^{\vee} \cap B O M_{p}^{-1}} \alpha_{p q} \cdot X_{p q c t}-\sum_{q}^{P^{\wedge} \cap B O M_{p}^{-1}} \alpha_{p q} \cdot X_{q c t} .
$$

- Constraints (13): logical items in a customer facility,

$$
\begin{aligned}
& \forall p \in \bar{\Phi}, c \in C, t \in T \\
& \operatorname{Dem}_{p c t}=X_{p c t}-\sum_{q}^{P^{\vee} \cap B O M_{p}^{-1}} \alpha_{p q} \cdot X_{p q c t}-\sum_{q}^{P^{\wedge} \cap B O M_{p}^{-1}} \alpha_{p q} \cdot X_{q c t} .
\end{aligned}
$$

\subsubsection{Shipping constraints}

Constraints (14) and (15) mean that shipping quantities depend on the existence of facilities, shipping channels and items.

$$
\begin{aligned}
& Z_{u v} \leqslant X_{u} \text { and } Z_{u v} \leqslant X_{v} \quad \forall u \in U, v \in U-\{u\} \cup C, \\
& 0 \leqslant Z_{p u v t} \leqslant M_{\infty} \cdot Z_{u v} \text { and } Z_{p u v t} \leqslant M_{\infty} \cdot \lambda_{p} \quad \forall p \in \Phi, u \in U, v \in U-\{u\} \cup C, t \in T .
\end{aligned}
$$

\subsubsection{Inventory constraints}

Constraint (16) shows that the inventory depends on existence of physical items and production facilities.

$$
Y_{p u t} \leqslant M_{\infty} \cdot X_{u} \quad \text { and } \quad Y_{p u t} \leqslant M_{\infty} \cdot \lambda_{p} \quad \forall p \in \Phi, u \in U, t \in T .
$$

A key point of supply chain design is to evaluate whether the postponement of an item can be useful. Such a choice has an impact on the item's lead-time. Items with long lead-times require larger safety stocks. As a consequence, we model the safety stock of an item in a facility as a percentage of the flows during the item's lead-time. The flows under question will be the item internal production flow, the item supplying flow from other facilities, and the item delivering flow to customers. Thus:

$$
\left.Y_{p u t} \geqslant \operatorname{Cov}_{p u} \cdot C f_{p u} \cdot X_{p u t}+\sum_{v \neq u}^{U} C t_{p v u} \cdot Z_{p v u t}+\sum_{c}^{C} C t_{p u c} \cdot Z_{p u c t}\right) \quad \forall p \in \Phi, u \in U, t \in T .
$$

\subsubsection{Capacity constraints}

These constraints express that enough resource lines must be opened into existing facilities in order to manufacture the desired quantities.

$$
\sum_{p}^{P_{r}} X_{p u t} \cdot \text { URes }_{r p} \leqslant D \cdot L P O_{r u} \leqslant D \cdot \operatorname{Max} L P_{r u} \cdot X_{u} \quad \forall r \in R, u \in U, t \in T .
$$




\subsubsection{Binary variable constraints}

$$
\begin{aligned}
& X_{u}, Z_{u v}, \lambda_{p} \in\{0,1\}, L P O_{r u} \in N \quad \forall u \in U, v \in U \cup C, \forall p \in P \\
& \quad \text { and } \lambda_{p q} \in\{0,1\} \quad \forall q \in P^{\vee}, p \in B O M_{q} .
\end{aligned}
$$

\section{Experimental evaluation}

Some experiments were conducted on the problem of a wiring harness supplier of a car manufacturer.

The wiring harness system connects all the electrical components of a car. It is made of wires, connectors, relays, fuses, computers and is distributed all over the car. It is concerned with all the electrical or electronic functions, which are more than fifty. Consequently, the potential functional diversity is extremely large.

In practice, a restricted list of versions is proposed to the customers. But each version can be customized by adding optional components. For a given set of functional requirements (a market segment) many design choices exist: one or several computers (in the front, for the motor, in the dashboard, in the back of the car) connected or not with a bus, the use of relays or the connection of a function to a computer, where to place connectors so that the wiring harness can be decomposed according to the location (door, motor, roof, front, back) or functionally (ABS, Air bag, Air conditioning). This means that many variants can be designed for a same market segment.

In this experiment the optional functions are not concerned. The generic bill-of-materials (G-BOM) is depicted in Fig. 8 and results from a market segment strategy. Variants are assembled from basic components (components 1 up to 48) and from computers (components 49, 50 and 51).

One key interest was to limit the number of versions of computers that are expensive and produced in long runs. Therefore, several variants were designed per market segment using different computer components. Note that some other components are also common to several variants.

The various combinations of choices relevant to the "OR" nodes of this generic bill-of-materials cover 48 product families. The optimization model must choose one of these product families while minimizing the total cost of the supply chain.

Car manufacturers require that their first rank suppliers use synchronous facilities in order to achieve a synchronous delivery. But the suppliers decide about the activities assigned to these synchronous facilities: inventory, assembly of the finished products, and component manufacturing. Wiring harness assembly

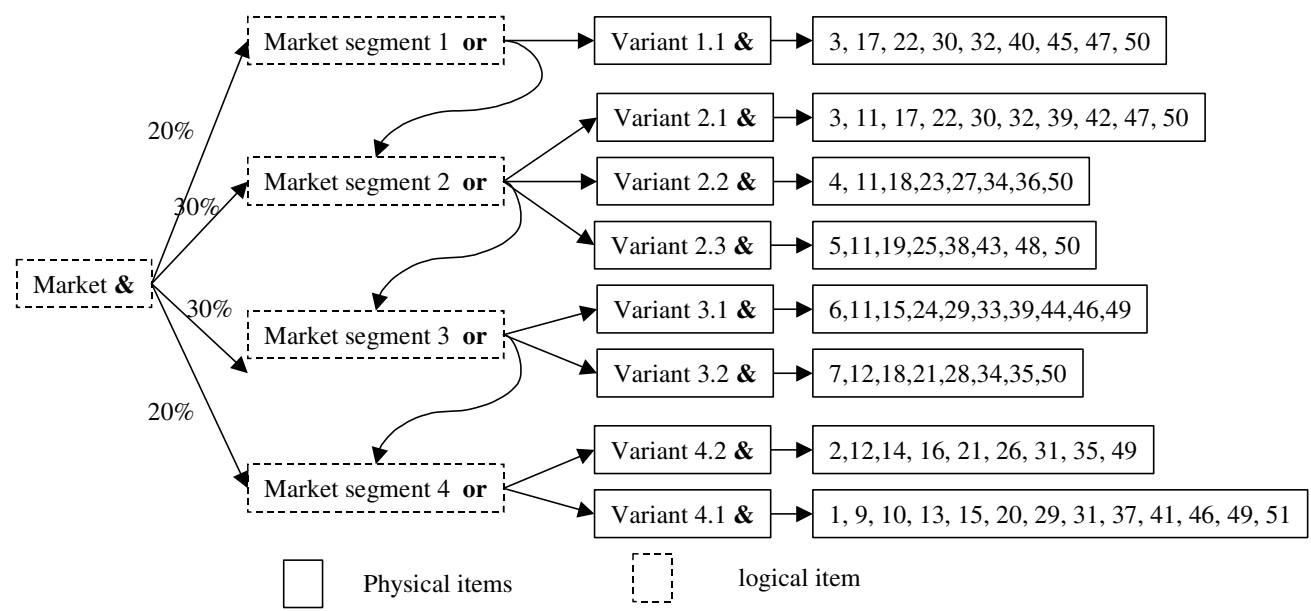

Fig. 8. A G-BOM for the application case. 
requires a lot of manpower because the assembly process of wires cannot be automated. Consequently, it seems interesting to investigate manufacturing and assembly possibilities in low cost countries. However, this is less interesting for computer manufacturing facilities as they require some investments in high technology facilities and consequently a skilled workforce.

Fig. 9 depicts the supply chain that has been studied. Some of the facilities were not initially opened and one interest of the approach was to evaluate if opening them could reduce the supply chain total cost.

The supply chain gathers: 4 synchronous facilities, each one dedicated to a customer, 7 manufacturing facilities with 3 dedicated to computers and 4 to components.

The previous model has been coded in $\mathrm{C}++$ with an Ilog-Cplex 6.5 library. With a demand expressed on 8 periods, the linear problem gathers 30,849 constraints, 20,073 real variables and 559 integer variables. It has been solved with a SUN UltraSPARC/143MHz/192Mo-RAM in 744 seconds. The solution is depicted in Fig. 10 for the product family and in Fig. 11 for the supply chain layout.

The same experiment has been processed with some simplified generic bill-of-materials, meaning that each generic bill-of-materials represents a smaller combinatory. This is achieved by fixing choices on some of the "OR" BOM nodes while keeping the same optimal solution. Computation time decreases with the combinatory, as shown in Fig. 12, until the duration reaches 115 seconds when combinatory equals 1 . For

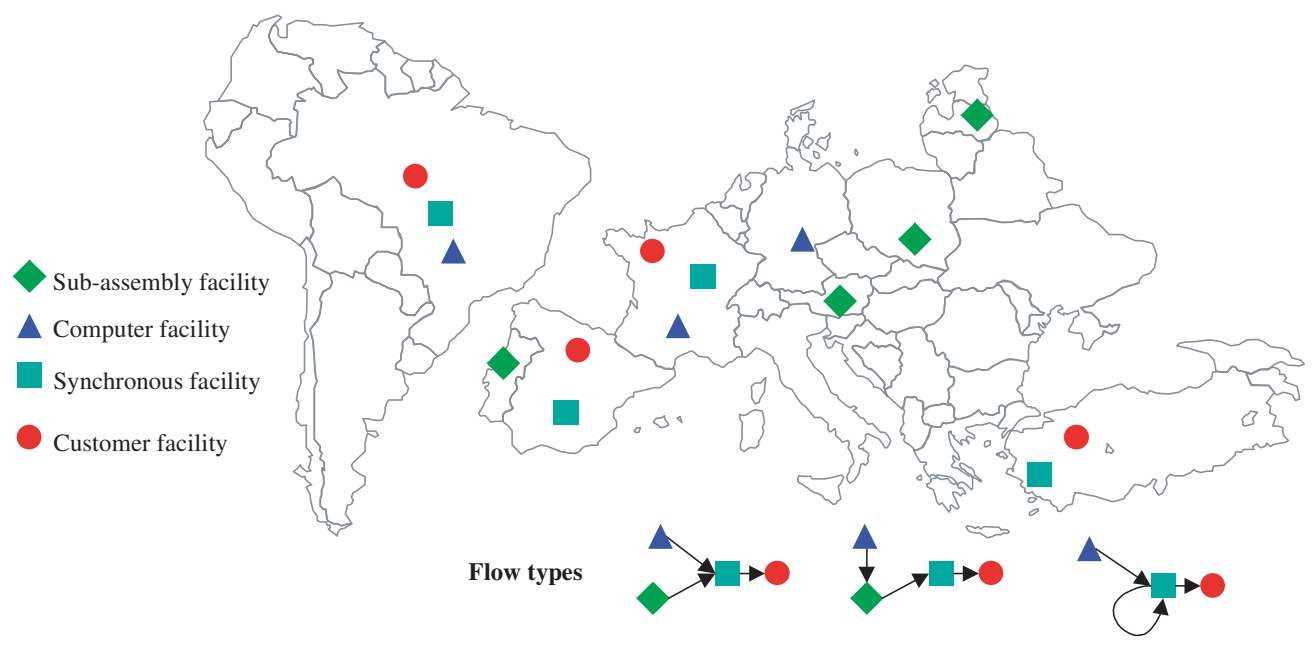

Fig. 9. The supply chain that must be designed.

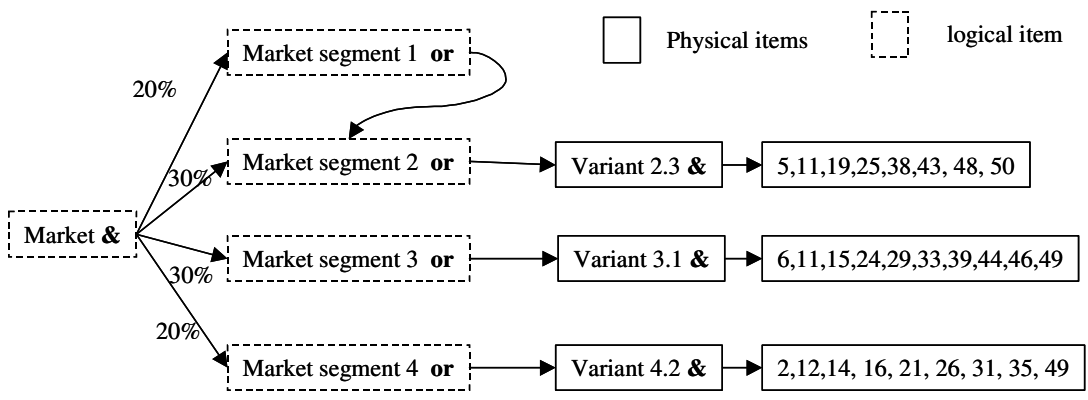

Fig. 10. Product family solution. 


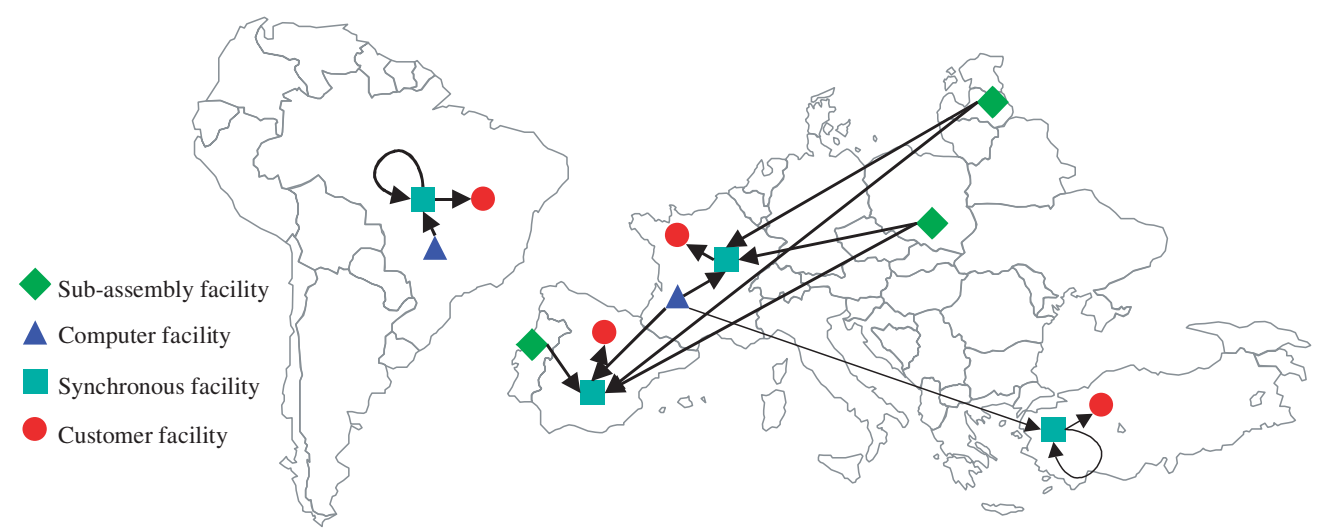

Fig. 11. The solution of the supply chain layout.

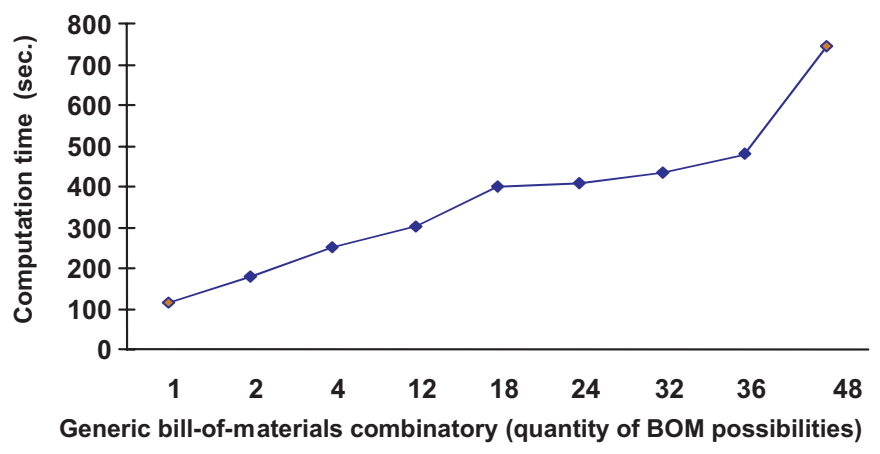

Fig. 12. Computational times versus the combinatory of the generic BOM.

this last case, the generic bill-of-materials corresponds with the bill-of-materials of the optimal solution. Thus, it is better to solve the problem once ( 744 seconds) than solving 48 problems (115 seconds each).

Another interest of the proposed model is to investigate the influence of the fixed cost of existence of variants on the diversity level of the product family. The diversity level is defined by the number of variants of the product family. In order to do so, a set of experiments has been made in which the variant existence costs are multiplied by a coefficient between 0.01 and 150 . The graphs of Fig. 13 show the sensibility of the diversity level with respect to the fixed cost of existence of variants.

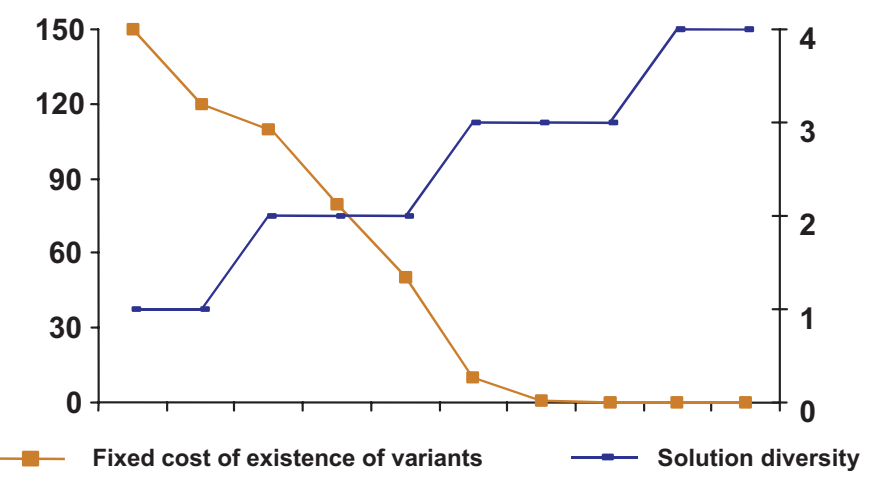

Fig. 13. Sensibility of the diversity with respect to variant existence cost. 


\section{Conclusion}

In the goal of taking into account demand and product diversity, the two-step approach presented in this paper allows to design a set of product families then to identify one product family and the layout of its supply chain while minimizing the cost compromise "over equipment cost/references management cost".

It has been shown that the result of the first step could be modeled as a generic bill-of-materials (GBOM). Compared with a classical bill-of-materials using only physical articles and "AND" nodes, this G-BOM introduces "logical articles" and "OR" nodes that allow to represent a set of product families that fulfils a total demand characterized by a large diversity.

A mixed integer linear programming (MILP) model is introduced that enables to identify the product family and its relevant supply chain, while optimizing a cost function. Compared to classical Global Supply Chain Models, the proposed constraints and flow conservation equations permit to take into account the proposed G-BOM. Some experiments showed that it was possible to solve this model in a reasonable amount of time and the interest of the G-BOM formulation in terms of processing time.

Many extensions have been introduced in global supply chain models such as financial cost (duties, taxes, exchange rates) or scale economies or choices of technological manufacturing systems or demand instability. All these extension needs to be integrated with the G-BOM one.

The proposed approach takes into account three kinds of diversity:

- the market diversity thanks to requirements and service levels,

- the product diversity thanks to components and variants,

- the supply chain layout diversity thanks to logistics aspects.

In current industrial context where demand, design activities and facilities are more and more worldwide distributed. The proposed elements can be considered as a step towards a global design approach. The example that illustrates our proposition comes from the automotive industry, which is at the intersection of product diversity and logistic aspects. Nevertheless, this kind of problem can be met in many other industrial domains like for example electrical appliance, PC industry, machine tools or electronic systems.

\section{References}

[1] D.M. Anderson, Agile Product Development for Mass Customisation, McGraw-Hill, 1998.

[2] B.C. Arntzen, G.G. Brown, T.P. Harrison, L.L. Trafton, Global supply chain management at digital equipment corporation, Interfaces 25 (1) (1995) 69-93.

[3] M.A. Cohen, H.L. Lee, Manufacturing strategy: Concepts and methods, in: P.R. Kleindorfer (Ed.), The Management of Productivity and Technology in Manufacturing, Plenum, New York, 1985.

[4] M.A. Cohen, H.L. Lee, Strategic analysis of integrated production-distribution systems: Models and methods, Operations Research 36 (2) (1988) 216-228.

[5] M.A. Cohen, H.L. Lee, Resource deployment analysis of global manufacturing and distribution networks, Journal of Manufacturing and Operations Management 2 (1989) 81-104.

[6] M.H. Cole, Service considerations and the design of strategic distribution systems, Ph.D. thesis, School of Industrial and Systems Engineering, Georgia Institute of Technology, Atlanta, 1995.

[7] G. Erixon, Design for modularity, in: G.Q. Huang (Ed.), Design for X: Concurrent Engineering Imperatives, Chapman \& Hall, 1996, pp. 356-379.

[8] A.M. Geoffrion, G.W. Graves, Multicommodity distribution system design by benders decomposition, Management Science 20 (5) (1974) 822-844.

[9] A.M. Geoffrion, G.W. Graves, S.J. Lee, Strategic distribution system planning: A status report, in: A.C. Hax (Ed.), Studies in Operations Management, North-Holland, Amsterdam, 1978, pp. 179-204.

[10] A.M. Geoffrion, G.W. Graves, S.J. Lee, A management support system for distribution planning, INFOR 20 (4) (1982) $287-314$. 
[11] R. Ganeshan, E. Jack, M.J. Magazine, P. Stephens, A taxonomic review of supply chain management research, in: Quantitative Models for SCM, Kluwer Academic, Boston, 1999, pp. 839-879.

[12] M. Goetschalckx, Strategic network planning, in: H. Stadler, C. Kilger (Eds.), Supply Chain Management and Advanced Planning, Springer, Heidelberg, Germany, 2000.

[13] M. Goetschalckx, S. Ahmed, A. Shapiro, T. Santoso, Designing flexible and robust supply chains, in: International Conference on Industrial Engineering and Production Management, Quebec City, Canada, 2001.

[14] K. Hadj-Hamou, E. Caillaud, J. Lamothe, M. Aldanondo, Knowledge for product configuration, in: International Conference on Engineering Design, Glasgow, 2001.

[15] G.Q. Huang, Design for X: Concurrent Engineering Imperatives, Chapman \& Hall, 1996.

[16] H.L. Lee, Design for supply chain management: Concept and examples, in: R. Sarin (Ed.), Perspectives in Operations Management, Kluwer Academic Publishers, Boston, MA, 1993, pp. 835-847.

[17] H.L. Lee, Product universality and design for supply chain management, Production Planning and Control 6 (3) (1995) $270-277$.

[18] H.L. Lee, C. Billington, Designing products and processes for postponement, in: S. Dasu, C. Eastman (Eds.), Management of Design Engineering and Management Perspectives, Kluwer Academic Publishers, Boston, MA, 1994, pp. 105-122.

[19] H.L. Lee, C.S. Tang, Variability reduction through operations reversal, Management Science 44 (2) (1998) 162-172.

[20] C.A. Lucas, G. Mitra, S. Mirhassani, Supply chain planning under uncertainty, in: E. Hadjiconsrantinou (Ed.), Quick Response in the Supply Chain, Springer, 1999.

[21] A. Martel, U. Vankatadri, Optimizing supply network structures under economies of scale, in: International Conference on Industrial Engineering and Production Management, Glasgow, 1999.

[22] G. Pahl, W. Beitz, Engineering Design: A Systematic Approach, second ed., Springer-Verlag, London, 1996.

[23] M. Paquet, A. Martel, G. Desaulniers, Including technology selection decisions in manufacturing network design models, in: International Conference on Industrial Engineering and Production Management, Quebec City, Canada, 2001.

[24] B.J. Pine II, Mass Customization: The New Frontier in Business Competition, Harvard Business School Press, Boston, 1993.

[25] B. PrasadConcurrent Engineering Fundamentals: Integrated Product and Process Organization, vol. 1, Prentice Hall, 1996.

[26] R.I. Van Hoek, The rediscovery of postponement: A literature review and directions for research, Journal of Operations Management 19 (2001) 161-184.

[27] C.J. Vidal, M. Goetschalckx, Strategic production distribution models: A critical review with emphasis on global supply chain models, European Journal of Operational Research 98 (1998) 1-18. 